\title{
Rapid optimization of drug combinations for the optimal angiostatic treatment of cancer
}

\author{
Andrea Weiss ${ }^{1,2} \cdot$ Xianting Ding $^{3} \cdot$ Judy R. van Beijnum $^{2}$ • \\ Ieong Wong ${ }^{4}$ - Tse J. Wong ${ }^{2}$ Robert H. Berndsen ${ }^{1,2}$. \\ Olivier Dormond ${ }^{5} \cdot$ Marchien Dallinga $^{6} \cdot$ Li Shen $^{7} \cdot$ Reinier O. Schlingemann $^{6}$. \\ Roberto Pili ${ }^{7}$ C Chih-Ming $\mathrm{Ho}^{4}$ - Paul J. Dyson ${ }^{1}$ - Hubert van den Bergh ${ }^{1}$ • \\ Arjan W. Griffioen ${ }^{2} \cdot$ Patrycja Nowak-Sliwinska $^{1,2}$
}

Received: 25 January 2015/Accepted: 13 March 2015/Published online: 1 April 2015

(C) The Author(s) 2015. This article is published with open access at Springerlink.com

\begin{abstract}
Drug combinations can improve angiostatic cancer treatment efficacy and enable the reduction of side effects and drug resistance. Combining drugs is non-trivial due to the high number of possibilities. We applied a feedback system control (FSC) technique with a population-based stochastic search algorithm to navigate through the large parametric space of nine angiostatic drugs at four concentrations to identify optimal low-dose drug combinations. This implied an iterative approach of in vitro testing of endothelial cell viability and algorithm-based analysis. The optimal synergistic drug combination, containing erlotinib, BEZ-235 and RAPTA-C, was reached in
\end{abstract}

Electronic supplementary material The online version of this article (doi:10.1007/s10456-015-9462-9) contains supplementary material, which is available to authorized users.

Patrycja Nowak-Sliwinska

Patrycja.Nowak-Sliwinska@epfl.ch

1 Institute of Chemical Sciences and Engineering, Swiss Federal Institute of Technology (EPFL), 1015 Lausanne, Switzerland

2 Department of Medical Oncology, VU University Medical Center, 1007 MB Amsterdam, The Netherlands

3 Med-X Research Institute, School of Biomedical Engineering, Shanghai Jiao Tong University, Shanghai 200030, China

4 Department of Mechanical and Aerospace Engineering, University of California, Los Angeles, CA 90095, USA

5 Department of Visceral Surgery, Centre Hospitalier Universitaire Vaudois, 1011 Lausanne, Switzerland

6 Ocular Angiogenesis Group, Departments of Ophthalmology and Cell Biology and Histology, Academic Medical Center, 1100 DD Amsterdam, The Netherlands

7 Department of Medicine, Roswell Park Cancer Institute, Buffalo, NY 14263, USA a small number of iterations. Final drug combinations showed enhanced endothelial cell specificity and synergistically inhibited proliferation $(p<0.001)$, but not migration of endothelial cells, and forced enhanced numbers of endothelial cells to undergo apoptosis $(p<0.01)$. Successful translation of this drug combination was achieved in two preclinical in vivo tumor models. Tumor growth was inhibited synergistically and significantly ( $p<0.05$ and $p<0.01$, respectively) using reduced drug doses as compared to optimal single-drug concentrations. At the applied conditions, single-drug monotherapies had no or negligible activity in these models. We suggest that FSC can be used for rapid identification of effective, reduced dose, multi-drug combinations for the treatment of cancer and other diseases.

Keywords Anti-angiogenesis - Combination therapy · Drug-drug interactions $\cdot$ Feedback system control $\cdot$ Search algorithm

\section{Introduction}

Anti-angiogenic therapies are routinely used in the treatment of various cancers [1-3]. Their contribution to the prolongation of patient survival, however, is often limited mainly due to disease and patient heterogeneity [4, 5], toxicity [6], induction of metastasis [7] and drug resistance $[8,9]$. Redundancy of growth factor signaling pathways makes angiogenesis a robust physiological function [10, 11], where targeting multiple pathways with drug combinations may be necessary for efficient therapy [12]. Although difficult to predict, in such drug combinations one may encounter synergistic, additive or antagonistic interactions between drugs. Synergistic interactions can 
lead to effective angiogenesis inhibition at reduced doses as compared to single-drug therapies. Combination strategies may thus lead to enhanced efficacy $[13,14]$ with limited side effects [15] and reduced probability of developing drug resistance $[16,17]$.

Combinations of anti-angiogenic drugs have often resulted in significant clinical toxicity [18], even when designed to target complementary pathways [19]. This is because drugs to be combined are frequently selected based on their success as single agents [20] and tend to be used in combination at their maximum tolerated single agent doses, thus increasing the risk of toxicity and resistance [21]. When trying to identify an optimal combination starting from, for instance, 10 drugs at 5 doses, one will have to test nearly 10 million $\left(5^{10}\right)$ combinations. To overcome this challenge, we employed a feedback system control (FSC) technique to rapidly identify the most powerful drug combinations with minimal experimental effort [22] (Supplementary Methods). In combination with the differential evolution (DE) algorithm [23], an iterative approach of experimental testing in an endothelial cell viability assay and mathematical analysis (a process of selection, where only the permutations which improve the system's response are maintained) drove the system to converge toward an optimal solution, i.e., maximal inhibition of endothelial cell growth. Although others have tried to optimize drug combinations [24-27], see Supplementary Methods, the advantage of our approach is that FSC is phenotypically driven, i.e., no mechanistic information is required in order to rapidly identify experimentally verifiable optimal drug combinations [22].

The aim of the present study was to find an optimal lowdose, synergistic anti-angiogenic drug combination using the FSC technology, and to validate this drug combination in preclinical tumor models. The FSC technique, together with a second-order linear regression model to allow for elimination of less effective drugs, resulted in the identification of the optimal low-dose combination containing erlotinib (EGFR inhibitor [28]), RAPTA-C (histone inactivator [29]) and BEZ-235 (a dual PI3K/mTOR inhibitor [30]). This final drug combination synergistically inhibited ECRF24 viability, while having minimal effects on nonendothelial cell types. We successfully translated this in vitro optimized drug combination to inhibit tumor growth in two preclinical tumor models.

\section{Materials and methods}

\section{Cell viability, migration and apoptosis assay}

Cell viability and migration assays were performed as previously described. Cells were seeded in a 96-well culture plate at a density of $2.5-10 \times 10^{3}$ cells/well. Cells were incubated with drugs for $72 \mathrm{~h}$ (for drug acquisition and cells and culture conditions, see Supplementary Methods). Drugs were premixed in culture medium and applied at the doses provided in Table 1 . Cell viability was assessed using the CellTiter-Glo luminescence assay (Promega, Madison, WI, USA). For migration assays, ECRF24 and 786-O were seeded in 96-well cell culture plates $\left(3 \times 10^{4}\right.$ cells/well) $24 \mathrm{~h}$ prior to making the scratch (Peira Scientific Instruments, Beerse, Belgium). Drugs were premixed in culture medium and applied at doses indicated in Supplementary Fig. S2A. Images were automatically captured on a Leica DMI3000 microscope (Leica, Rijswijk, Netherlands) at $5 \times$ magnification with Universal Grab 6.3 software (DCILabs, Keerbergen, Belgium). Scratch sizes were determined at $t=0 \mathrm{~h}$ and $t=7 \mathrm{~h}$ using Scratch Assay 6.2 (DCILabs), and values reported represented the absolute closure of the scratch (initially subtracting the final scratch area). Apoptosis was measured after drug exposure, trypsinization and incubation with propidium iodide (PI, $20 \mu \mathrm{g} / \mathrm{ml}$ ) in DNA extraction buffer [31], by flow cytometry. Tip cells were flow cytometrically quantified by CD34 [32] staining, and morphology was studied in vivo using the CAM assay [33] (see Supplementary Methods).

\section{The feedback system control (FSC) technique and data modeling}

The FSC technique was employed as previously described $[34,35]$. FSC was implemented using the DE algorithm,

Table 1 Drug dose values used in the in vitro cell viability assays

\begin{tabular}{llccl}
\hline & Dose $(\mu \mathrm{M})$ & & & \\
\cline { 2 - 5 } & Drug & $3\left(\mathrm{ED}_{10}\right)^{\mathrm{a}}$ & $2\left(\mathrm{ED}_{5}\right)^{\mathrm{b}}$ & $1\left(\mathrm{ED}_{0}\right)^{\mathrm{c}}$ \\
\hline 1. & Anginex & $1.80^{\mathrm{d}}$ & 0.76 & 0.13 \\
2. & Bevacizumab & 15.00 & 10.00 & 1.00 \\
3. & Axitinib & 1.00 & 0.30 & 0.01 \\
4. & Erlotinib & 2.00 & 0.50 & 0.10 \\
5. & Anti-HMGB1 Ab & 0.17 & 0.09 & 0.02 \\
6. & Sunitinib & 0.50 & 0.10 & 0.05 \\
7. & Anti-vimentin Ab & 0.26 & 0.17 & 0.09 \\
8. & RAPTA-C & 5.00 & 1.00 & 0.05 \\
9. & BEZ-235 & 0.005 & 0.001 & 0.0005 \\
\hline
\end{tabular}

${ }^{a}$ Dose 3, representing $\mathrm{ED}_{10}$, is the dose where $10 \%$ of the maximal response was observed

${ }^{\mathrm{b}}$ Dose 2, representing $\mathrm{ED}_{5}$, is the dose where $5 \%$ of the maximal response was observed

c Dose 1, representing $\mathrm{ED}_{0}$, is the dose representing half the maximal concentration where no effect was observed

${ }^{\mathrm{d}}$ Concentrations throughout the table are in $\mu \mathrm{M}$ 
and two separate optimizations were performed with the cellular outputs of ECRF24 cell viability (proliferation) and migration assays. Nineteen drug combinations were tested per iteration, and 10 iterations were performed in each optimization until a plateau in the best output value was reached. For dilutions and culture conditions, see Supplementary Methods. The cells were incubated in $50 \mu \mathrm{l}$ of each combination for $72 \mathrm{~h}$ in the viability assay or for $7 \mathrm{~h}$ in the migration assay.

Second-order linear regression models were generated using the data obtained from each optimization. Data were modeled using real concentration values, and both concentration values and cell viability output data were transformed using the $z$ score function in MATLAB. For detailed description, see Supplementary Methods.

\section{Human ovarian carcinoma grown on the chicken chorioallantoic membrane (CAM)}

Human ovarian carcinoma tumors were implanted on the CAM as previously described [36]. On embryo development day (EDD) 7, $1 \times 10^{6}$ A2780 carcinoma cells were prepared as a spheroid in a $25-\mu 1$ hanging drop and were transplanted onto the CAM surface $3 \mathrm{~h}$ after preparation. Treatment began 3 days after tumor implantation (EDD10) when vascularized tumors were visible. Drug combinations were freshly prepared and administered as a $20-\mu l$ intravenous injection. Treatment was performed twice, and tumor growth was monitored and measured daily (volume $=$ width $^{2} \times$ length $\left.\times 0.52\right)$.

\section{Colorectal carcinoma xenograft model}

Female Swiss nu/nu mice aged 6-8 weeks were obtained from Charles River (weight 20-30 g). Mice were inoculated in the right flank with $100 \mu \mathrm{l}$ DMEM with 1 million LS174T cells. LS174T cells were obtained from ECACC, Salisbury, UK (authentication by STR PCR), and were used within 6 months of resuscitation. Palpable tumors were present within 3-5 days, at which time treatment was initiated. Mice were treated daily by oral gavage and i.p. injection as indicated (Table 2) and were monitored daily for tumor size and body weight (see Supplementary Methods).

\section{Immunohistochemistry}

CD31 staining (SZ31, Dianova, Hamburg, Germany) was performed using donkey anti-rat biotinylated secondary antibodies (Jackson, Suffolk, UK) and streptavidin-HRP (Dako, Glostrup, Denmark) and visualized by 3,3'-diaminobenzidine (DAB, see Supplementary Methods).

\section{Statistical analysis}

Values are given as mean values \pm SD. Statistical analysis was performed using a two-sided student's $t$ test and the two-way ANOVA assay. $* p<0.05$ and $* * p<0.01$ were considered statistically significant.

\section{Results}

\section{Selection of drug combinations by the FSC technique}

Nine drugs targeting a broad spectrum of endothelial cell signaling pathways (Supplementary Fig. S1; Supplementary Methods) were selected for FSC-based screening (Fig. 1a): anginex (1), bevacizumab (2), axitinib (3), erlotinib (4), anti-HMGB1 Ab (5), sunitinib (6), antivimentin Ab (7), RAPTA-C (8) and BEZ-235 (9). Singledrug dose-response curves were generated for both cell viability (example for sunitinib provided, Fig. 1b) and migration, using in vitro bioassays (Supplementary Fig. S2A). The optimization was carried out with each compound at four low doses. The highest concentration, dose 3 or $\mathrm{ED}_{10}$, was the dose where $10 \%$ of the maximal response was observed, dose 2 or $\mathrm{ED}_{5}$, where $5 \%$ of the maximal response was observed, dose 1 or $\mathrm{ED}_{0}$, represented half the maximal dose where no effect was observed, and dose 0 , where no drug was present (Table 1). Starting from randomly selected drug combinations (Fig. 1a, yellow arrow), the FSC technique implements an algorithm-guided closedloop feedback search to iteratively optimize the results of an in vitro cell assay (blue arrows). The box plot in Fig. 1c provides the median and interquartile ranges of the output results of the drug combinations identified by the end of each iterative cycle of the FSC optimization. After 10 iterations of 19 drug mixtures, the optimization goal was reached, i.e., no further improvement of the lowest output efficacy could be achieved, indicating that the maximum activity (approx. $70 \%$ inhibition) had been reached.

The data obtained from this optimization process were used to build a second-order stepwise linear regression model [37] (Supplementary Methods) to determine the relative importance of the individual drugs. This model generated regression coefficients (Fig. 1d) corresponding to single-drug linear effects (left panel), two-drug pair-wise interaction effects (middle panel) and single-drug quadratic effects (right panel). Compounds with the largest negative regression coefficients, i.e., axitinib, erlotinib, RAPTA-C and BEZ-235, inhibited ECRF24 viability most effectively (Fig. 1d, green arrows). A regression model containing all regression coefficients (i.e., a non-stepwise linear regression model) is provided in Supplementary Fig. S2B. 
Table 2 Drug dose values used for in vivo assays

\begin{tabular}{|c|c|c|c|c|c|}
\hline \multirow[t]{2}{*}{ Treatment $^{\mathrm{b}}$} & \multicolumn{4}{|c|}{ Compounds $^{\mathrm{a}}$} & \multirow[t]{2}{*}{$\% \mathrm{CTRL} \pm \mathrm{SEM}^{\mathrm{c}}$} \\
\hline & $\begin{array}{l}3 \\
\text { (axitinib) }\end{array}$ & $\begin{array}{l}4 \\
\text { (erlotinib) }\end{array}$ & $\begin{array}{l}8 \\
\text { (RAPTA-C) }\end{array}$ & $\begin{array}{l}9 \\
\text { (BEZ-235) }\end{array}$ & \\
\hline \multicolumn{6}{|c|}{$C A M(\mu g / k g)$} \\
\hline I & 0 & 29 & 615 & 0.04 & $41 \pm 9.0$ \\
\hline II & 0 & 29 & 307 & 0.04 & $51 \pm 14$ \\
\hline VI & 0 & 2.9 & 307 & 0.04 & $32 \pm 4.0$ \\
\hline VII & 18 & 29 & 230 & 0.02 & $13 \pm 6.0$ \\
\hline VIII & 0 & 29 & 307 & 0 & $47 \pm 14$ \\
\hline \multicolumn{6}{|c|}{ Mice $(\mathrm{mg} / \mathrm{kg})$} \\
\hline VI & 0 & 15 & 40 & 10 & $24 \pm 14$ \\
\hline VIII & 0 & 5 & 40 & 0 & $84 \pm 16$ \\
\hline $4_{1}$ & 0 & 5 & 0 & 0 & $102 \pm 25$ \\
\hline $4_{2}$ & 0 & 15 & 0 & 0 & $94 \pm 34$ \\
\hline $4_{\mathrm{opt}}$ & 0 & 50 & 0 & 0 & $29 \pm 9.0$ \\
\hline $8_{2}$ & 0 & 0 & 40 & 0 & $90 \pm 17$ \\
\hline $8_{\text {opt }}$ & 0 & 0 & 100 & 0 & $58 \pm 9.0$ \\
\hline $9_{4}$ & 0 & 0 & 0 & 10 & $77 \pm 12$ \\
\hline $9_{\text {opt }}$ & 0 & 0 & 0 & 30 & $33 \pm 14$ \\
\hline
\end{tabular}

a Corresponding dose of each compound for single-drug and combination therapy. Compounds are represented as numbers $3,4,8$ or 9, representing axitinib, erlotinib, RAPTA-C or BEZ-235, respectively. Drug doses are provided in $\mu \mathrm{g} / \mathrm{kg}$ for the CAM model and $\mathrm{mg} / \mathrm{kg}$ in the mouse model

b Administered treatment, either a single drug represented by the drug number $(\mathbf{3}, \mathbf{4}, \mathbf{8}, \mathbf{9})$ and the dosage level indicated as a subscript, or drug combinations represented by the letters I-VIII

c The respective tumor growth inhibition efficacy represented as a percentage of the control $( \pm \mathrm{SEM})$
In a parallel approach, we also investigated the best drug combinations for ECRF24 migration inhibition. Even though single drugs generally showed a stronger response in the cell migration assay (Supplementary Fig. S2A), the process of migration was less affected, reaching a maximum effect of $40 \%$ inhibition in the given conditions (Supplementary Fig. S3B). The optimization of EC migration inhibition was not further pursued. Yet, regression analysis also revealed strong single-drug linear and quadratic contributions for erlotinib, RAPTA-C and BEZ235.

\section{Refined search leads to further optimized synergistic drug combinations}

Subsequently, a second FSC-based optimization was performed with the above-selected compounds, i.e., axitinib (3), erlotinib (4), RAPTA-C (8) and BEZ-235 (9), each now considered at five drug doses with a maximum activity of $25 \%$ at the highest dose (Fig. 2a; single-drug effects in Supplementary Fig. S3). The most effective combinations resulting in more than $50 \%$ inhibition of ECRF24 cell viability identified in the second screen are provided in Fig. 2a. The square icons represent the individual drug combinations, where the color and pattern can be used to identify the drug and its applied dosage. The strongest synergistic activity [i.e., combination index $(\mathrm{CI})<1$ ] was observed for combinations containing $\mathbf{4}+\mathbf{8}+\mathbf{9}$ (combinations labeled $\mathbf{I}, \mathbf{I I}, \mathbf{I V}, \mathbf{V}$, VI, Fig. 2a (remaining results in Supplementary Fig. S4) or only $\mathbf{4}+\mathbf{8}$ (labeled VIII). Two of the effective combinations identified, III and VII, showed antagonism $(\mathrm{CI}>1)$, and both contained axitinib (3).

Linear regression modeling of data showed the singledrug linear contributions of all compounds, as well as the single-drug quadratic effect of $\mathbf{4}$, to be significant (Fig. 2b, green arrows). Response surfaces (Fig. 2c) provide a visual representation of the relationship between the system output (EC viability) and the varying dose of only two drugs in the combination. Interestingly, surfaces containing 3 (bottom row) show that increasing the dose of $\mathbf{3}$ does not enhance the combination efficacy (red). These response surfaces show a relatively smooth response when doses of the given drugs are varied. This "smoothness" indicates that a moderate change in the dose of a single drug in the range of the experimental conditions near the optimal output investigated will most likely not result in a significant change in the output response. This implies a certain amount of "stability" in the optimal drug mixture which may facilitate its translation to different models. 

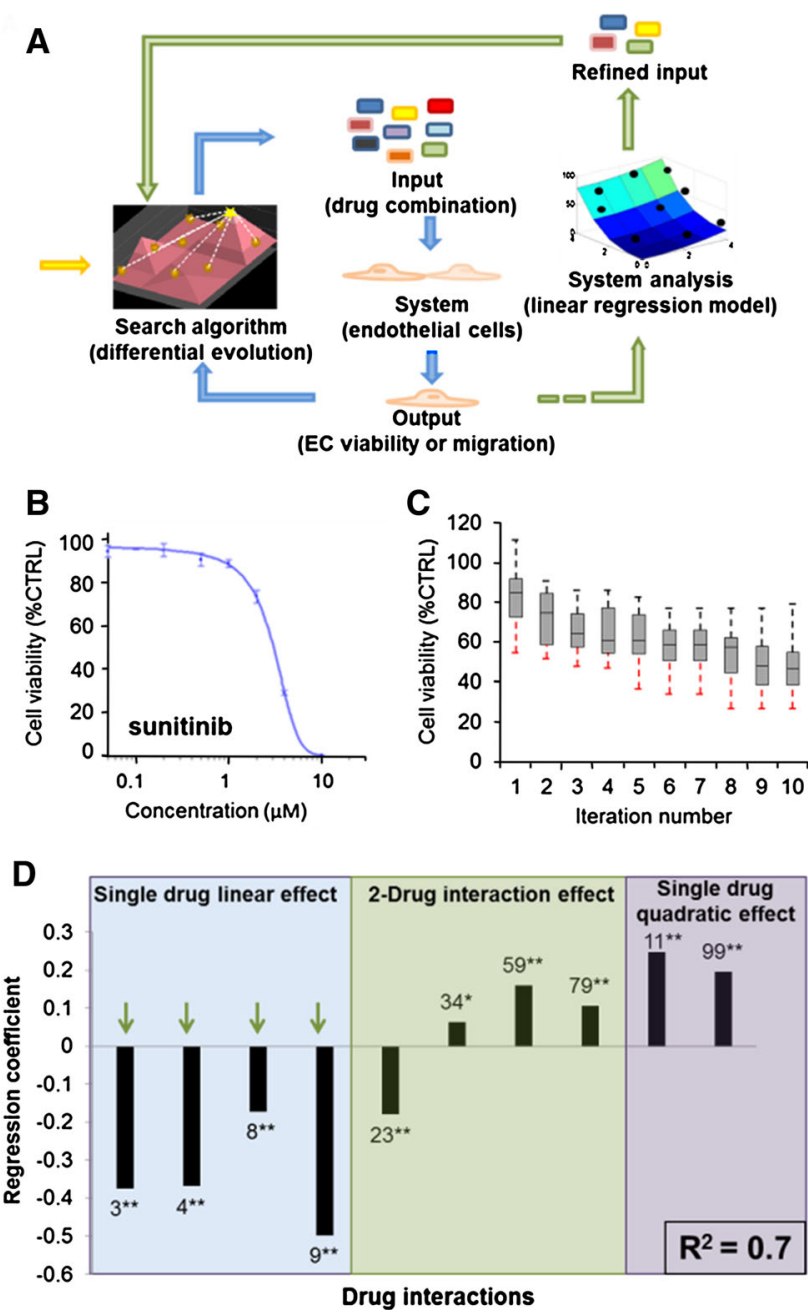

Fig. 1 Optimization of the inhibition of endothelial cell viability. a Schematic diagram of the FSC technique loop (blue arrow loop) and modeling (green arrow loop) used for in vitro drug optimization. FSC starts with randomly selected drug combinations (yellow arrow) and implements an algorithm-guided closed-loop feedback search to iteratively optimize the results of an in vitro cell assay (blue arrows). Once a plateau in the output is reached, the data obtained from the optimization are used to model the system, analyze drug interactions and eliminate certain drugs (green arrows). Using a refined set of drugs, the drug combination is again optimized with FSC (blue arrows). b Dose-response curve of sunitinib for cell viability bioassay. c Output results (in vitro EC viability, represented as a percentage of the control) for the 10 iterations of the FSC optimization performed. Box plots provide median and interquartile ranges of the cell response to the 19 best drug combinations identified by the end of each iterative cycle of the FSC optimization. Dotted lines, representing maximum and minimum (red) output values, showed no improvement in the best-optimized combination over iterations 8-10. d Regression coefficients obtained from the stepwise linear regression model generated with the data obtained from the optimization described above. The coefficient of determination $\left(R^{2}\right)$ is provided in the bottom right of the graph. Green arrows indicate single-drug contributions which significantly inhibit EC viability. * indicates $p$ value $<0.05$ and $* *$ indicates $p$ value $<0.01$. (Color figure online)

\section{Selected optimized drug combinations exhibit enhanced endothelial cell specificity}

The optimized drug combinations I-VIII (Fig. 2a) and corresponding single drugs were tested for viability of different cell types and shown in comparison with ECRF24 (Fig. 3a; Supplementary Fig. S5). The activity in ECRF24 was confirmed in primary ECs (HUVEC, Fig. 3a) and was much stronger than that of non-malignant cell types (adult human dermal fibroblasts (HDFa), human peripheral blood mononuclear cells (PBMCs) and tumor cells 786-O renal cell adenocarcinoma, HT-29 colorectal carcinomas, A2780 ovarian adenocarcinoma, LS174T colorectal adenocarcinoma and MDA-MB-231 breast adenocarcinoma), indicating an enhanced EC specificity. Combinations IVIII only modestly affected cell motility in ECRF24 and 786-O cell lines in a wound-healing or scratch assay (Fig. 3b). The effect of individual compounds and combinations on ECRF24 apoptosis induction was assessed based on the analysis of the DNA content by flow cytometry. Several combinations (II, III, V, VI, VII) induced apoptosis in 20-30 \% of ECRF24 cells (Fig. 3c).

Finally, the effect of combination VI on the inhibition of tip cells was assessed both in vitro and in vivo. FACS analysis shows a reduction in the number of $\mathrm{CD} 34^{+}$tip cells $[32,38,39]$ after treating HUVEC cultures in vitro with VI. Quantification of these results is provided in the bar graph as compared to single-drug treatments (Fig. 3d, top left). Additionally, CD34 ${ }^{+}$tip cells treated with VI present with a clearly different cellular organization of the actin fibers stained with phalloidin, as compared to control cells (Fig. 3d, top right), compatible with decreased migratory activity.

The inhibition of tip cells in vivo was shown in the chicken chorioallantoic membrane (CAM) model following vaso-occlusive Visudyne ${ }^{\circledR}$-photodynamic therapy (VPDT) [33]. In the control treatment group, vascular sprouts led by tip cells can be seen growing into the treated area $24 \mathrm{~h}$ after V-PDT treatment starting the revascularization of the tissue (Fig. 3d, bottom left, green arrows). A significant reduction in the number of sprouting tip cells can be seen in the group treated with VI following V-PDT (Fig. 3d, bottom right, red arrows).

\section{Successful translation of optimal drug combinations into in vivo cancer models}

A2780 cells were transplanted onto the chorioallantoic membrane of the chicken embryo and were allowed to grow forming vascularized tumors. Tumors were treated 

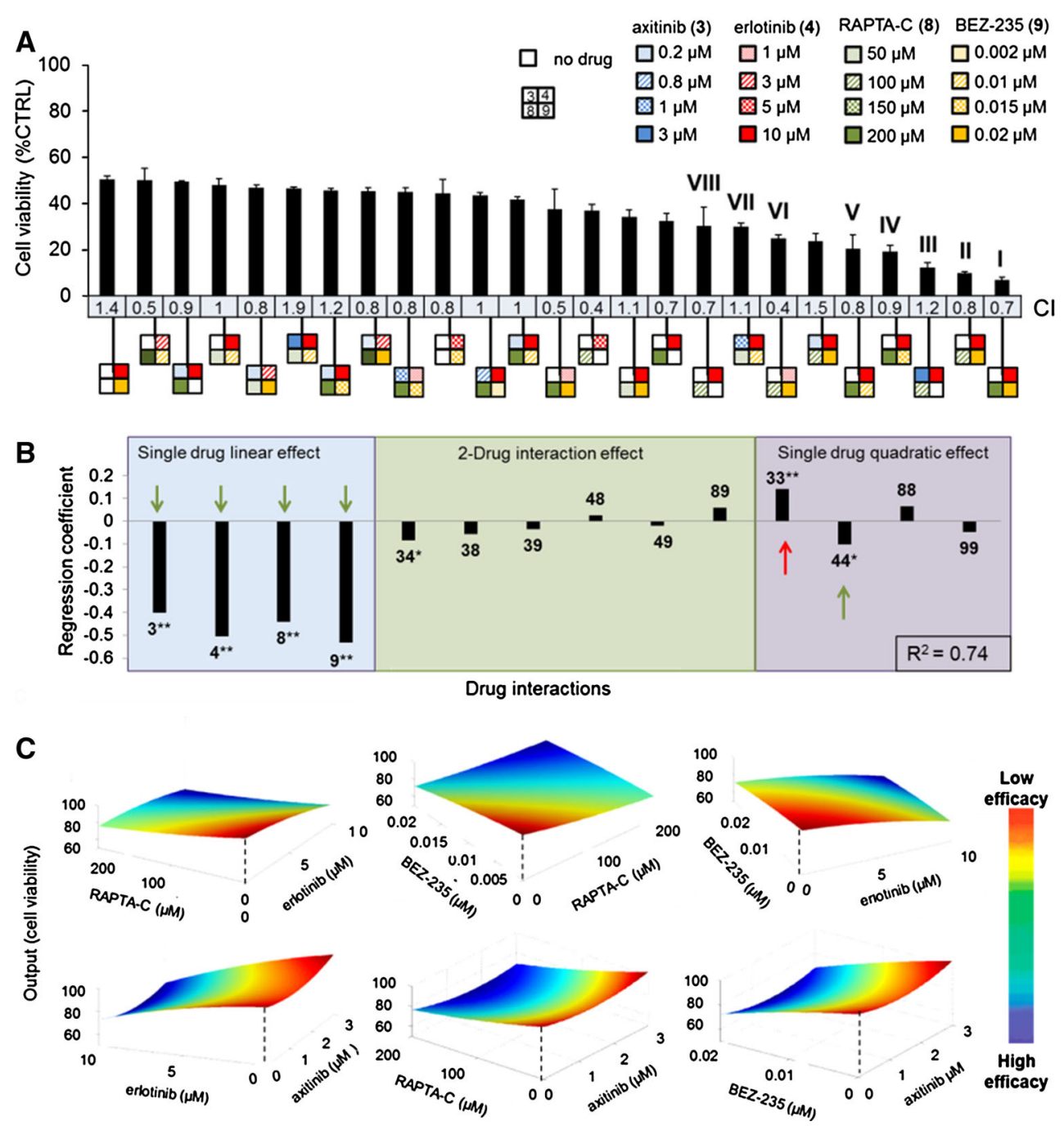

Fig. 2 Identification of the optimal four-drug combinations for the inhibition of ECRF24 viability. a Efficacy of the best combinations identified to inhibit ECRF24 viability, using the concentrations of each drug presented in the legend at the top right. Best-performing combinations resulting in $50 \%$ or more inhibition are shown, with their corresponding combination index $(\mathrm{CI})$ values calculated using CompuSYN $^{\circledR}$, indicating synergistic $(\mathrm{CI}<1)$, additive $(\mathrm{CI}=1)$ or antagonistic $(\mathrm{CI}>1)$ interactions. The square icons present the specific combinations, where each position in the square and color corresponds to a specific drug [i.e., axitinib (3) in blue, erlotinib (4) in red, RAPTA-C (8) in green and BEZ-235 (3) in yellow] and the concentrations (in $\mu \mathrm{M}$ ) of each compound are represented by the different patterns. The most promising combinations, labeled I-VIII,

with combinations I, II, VI, VII and VIII by intravenous injection on treatment days 1 and 2 (Fig. 4a, red arrows). Doses (subsequently identified by a subscript) were translated to this model maintaining the drug dose ratios and taking into account the single-drug efficacy in this model (Fig. 4b; Table 2, Supplementary Methods). Drug combination VII $\left(\mathbf{3}_{\mathbf{3}}+\mathbf{4}_{\mathbf{4}}+\mathbf{8}_{\mathbf{1}}+\mathbf{9}_{2}\right)$ synergistically (CI 0.66 ) inhibited tumor growth by $87 \%(* p<0.03$, Fig. 4a). Based on results in Fig. 3a, this activity could be due to the represent a mean of at least two independent experiments, with three replications each, and error bars represent the SEM. b Regression coefficients for the second-order linear regression model generated based on the data from the optimization of the refined four-drug combination. The green arrows indicate significant regression terms that inhibit cell viability, while the red arrow indicates terms that stimulates cell activity. c Response surfaces show the effect on the system output of varying the concentration of two drugs, while the concentration of the other two drugs remains fixed. Note the smoothness of the curves, indicating that moderate changes in the dosing of a single drug do not result in major output differences. $*$ indicates significance $p$ value $<0.05$ and ${ }^{* *} p$ value $<0.01$. (Color figure online)

dual action on both ECRF24 and A2780 cells. Combination VI $\left(\mathbf{4}_{1}+\mathbf{8}_{2}+\mathbf{9}_{4}\right)$ synergistically inhibited tumor growth by $68 \%$ (Fig. $4 \mathrm{a}, \mathrm{c}, * * p<0.002$, CI 0.34 ) through mainly anti-angiogenic activity (compare Fig. 3a). Of note, none of these doses inhibited tumor growth significantly when applied individually (Fig. 4b). As group VII experienced weight loss (Fig. 4d, **p $<0.004$ ), it was not further examined. Microvessel density (MVD) assessment (Fig. 4e) revealed that control tumors were well vascularized. MVD 


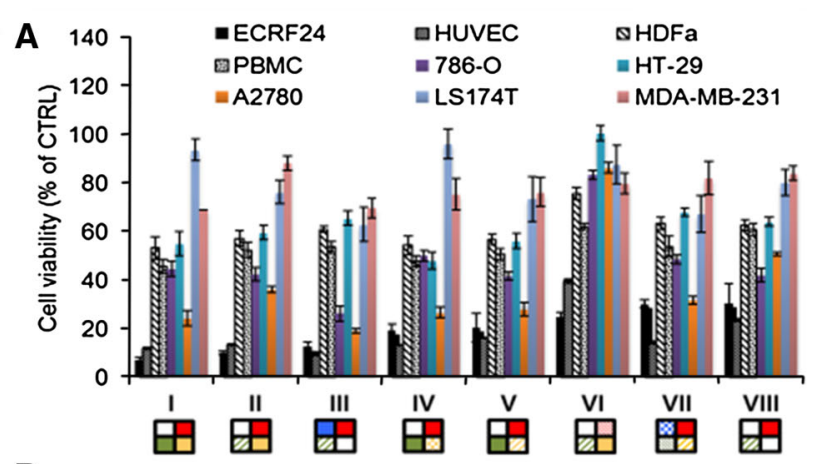

B
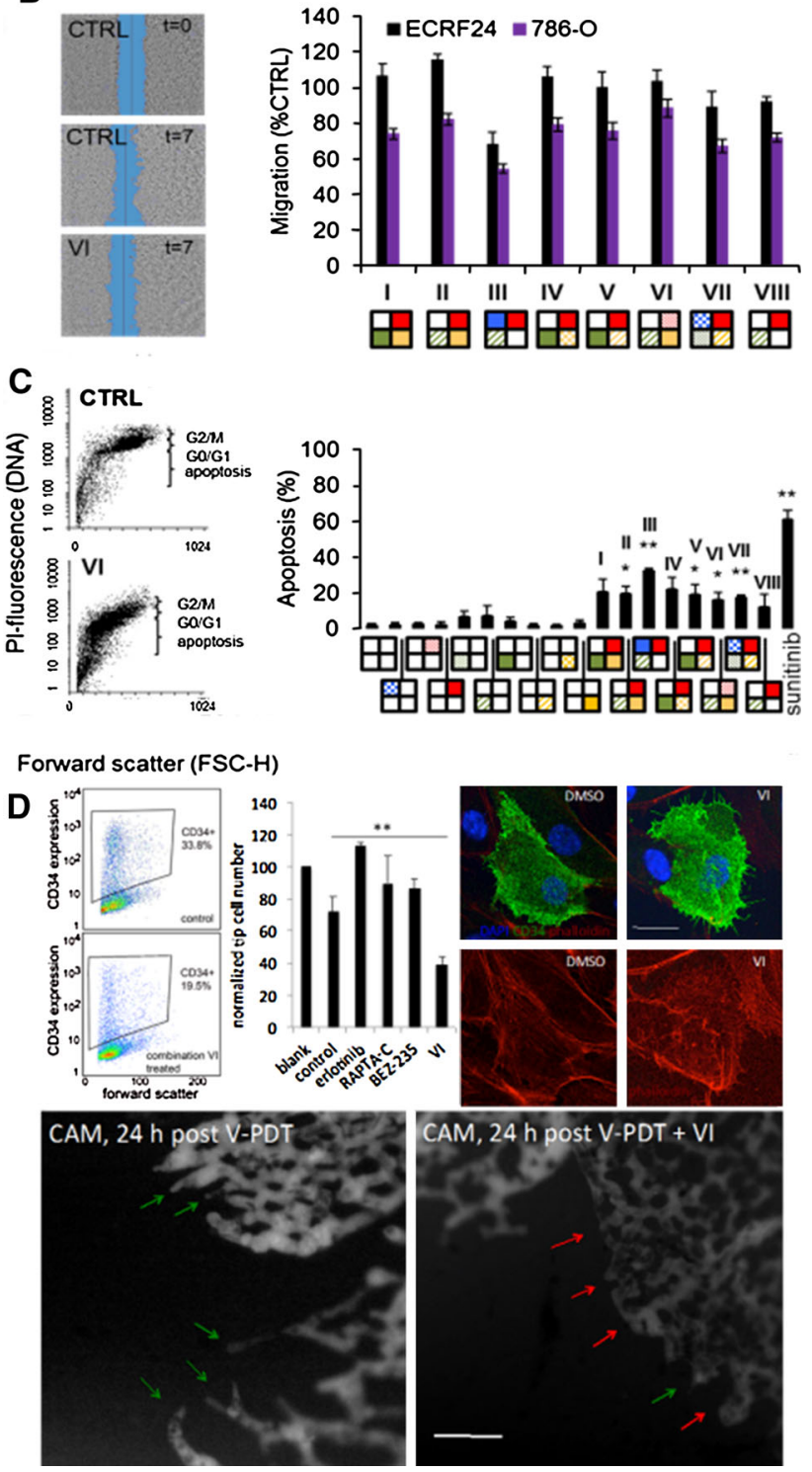

was 50-60\% lower in tumors treated with VI $(* * p<0.008)$ and VIII ( $* p=0.01$, Fig. 4f). Based on these data, VI was selected as the most promising combination.

Combinations VI and VIII were studied in athymic mice grafted with human LS174T colorectal adenocarcinoma. Drug doses were adapted to this model based on
4 Fig. 3 Validation of the best drug combinations. The effects of the most promising drug combinations (I-VIII from Fig. 2) were tested on the viability of the following non-malignant and cancerous cell lines: primary EC (HUVEC), adult human dermal fibroblasts (HDFa), human peripheral blood mononuclear cells (PBMCs) and five human tumor cell lines, i.e., A2780 ovarian adenocarcinoma, 786-O renal cell carcinoma, MDA-MB-231 breast adenocarcinoma and LS174T and HT-29 colorectal carcinomas (a) and on the migration of ECRF24 and 786-O cells (b). Images on the left show an example of migration assay, where a scratch is made in a cell monolayer at $t=0$ and the relative closure of this scratch is measured after $7 \mathrm{~h}$. c Effects of individual compounds and combinations on ECRF24 apoptosis induction. Images show the analysis of the DNA content by flow cytometry, after fixation of the cells in $70 \%$ ethanol, a DNA extraction step and staining with PI for cells in the control (CTRL) and combination VI group. * indicates significance $p$ value $<0.05$ and $* *$ indicates significance $p$ value $<0.01$ with student's $t$ test. Values represent the mean of at least two independent experiments with three replications each, and error bars represent the SEM. d Combination therapy VI inhibits tip cells in vitro and in vivo. FACS analysis show the decrease in $\mathrm{CD} 34^{+}$cells VI treated in HUVEC cultures, which is quantified in the bar graph and compared to single-drug treatments. CD $34^{+}$tip cells treated with VI present with a clearly different cellular organization of the actin fibers stained with phalloidin as compared to control cells, compatible with decreased migratory activity. Bar stands for $25 \mu \mathrm{m}$. The FITC-dextran fluorescence (FITC-dextran, $20 \mathrm{kDa}, 20 \mu \mathrm{l}, 25 \mathrm{mg} /$ $\mathrm{ml}$, Sigma-Aldrich) angiographies below show the chicken chorioallantoic membrane (CAM) capillary plexus at the edges of the Visudyne ${ }^{\circledR}$-photodynamic therapy (V-PDT; $5 \mathrm{~J} / \mathrm{cm}^{2}$ and $35 \mathrm{~mW} / \mathrm{cm}^{2}$ at $420 \pm 20 \mathrm{~nm}$ )-treated zones $24 \mathrm{~h}$ post-V-PDT, where the tip cells form the leading edge of the sprouting vasculature (green arrows). A major lack of sprouting tip cells (red arrows) is visible after treatment V-PDT with immediate of combination VI. (Color figure online)

single-drug tumor growth inhibition efficacy (Supplementary Methods). Mice were treated daily with vehicle (CTRL), VI $\left(\mathbf{4}_{2}+\mathbf{8}_{2}+\mathbf{9}_{4}\right)$ and VIII $\left(\mathbf{4}_{1}+\mathbf{8}_{2}\right)$ (Fig. 5a; Table 2). VI and VIII inhibited tumor growth significantly by $76 \pm 14 \%(* * p<0.0001, \mathrm{CI} 0.56)$ and $16 \pm 16 \%$ (CI 0.73 ), respectively (Fig. 5a). Drugs applied individually inhibited tumor growth only marginally, by $6 \%\left(\mathbf{4}_{2}\right), 10 \%\left(\mathbf{8}_{\mathbf{2}}\right)$ or $23 \%\left(\mathbf{9}_{\mathbf{4}}\right)(* p<0.013$, Fig. 5b). Interestingly, since the LS174T cell line was not sensitive to VI (Fig. 3a), effective tumor inhibition (Fig. 5a, c) was attributed to the inhibition of angiogenesis. MVD assessment indicated that VI suppressed angiogenesis by approximately $80 \%(* * p<0.001)$, compared with control tumors (Fig. 5d). No significant weight loss was recorded in either of the combinations tested (Fig. 5e). In contrast, individual compounds administered at optimal monotherapy doses, capable of effective tumor growth inhibition (Supplementary Fig. S6C) resulted in considerable body weight loss.

\section{Discussion}

Next to the identification of novel targets as well as endogenous and synthetic novel angiogenesis inhibitors [40, 41], the combination of therapies is globally seen as a 

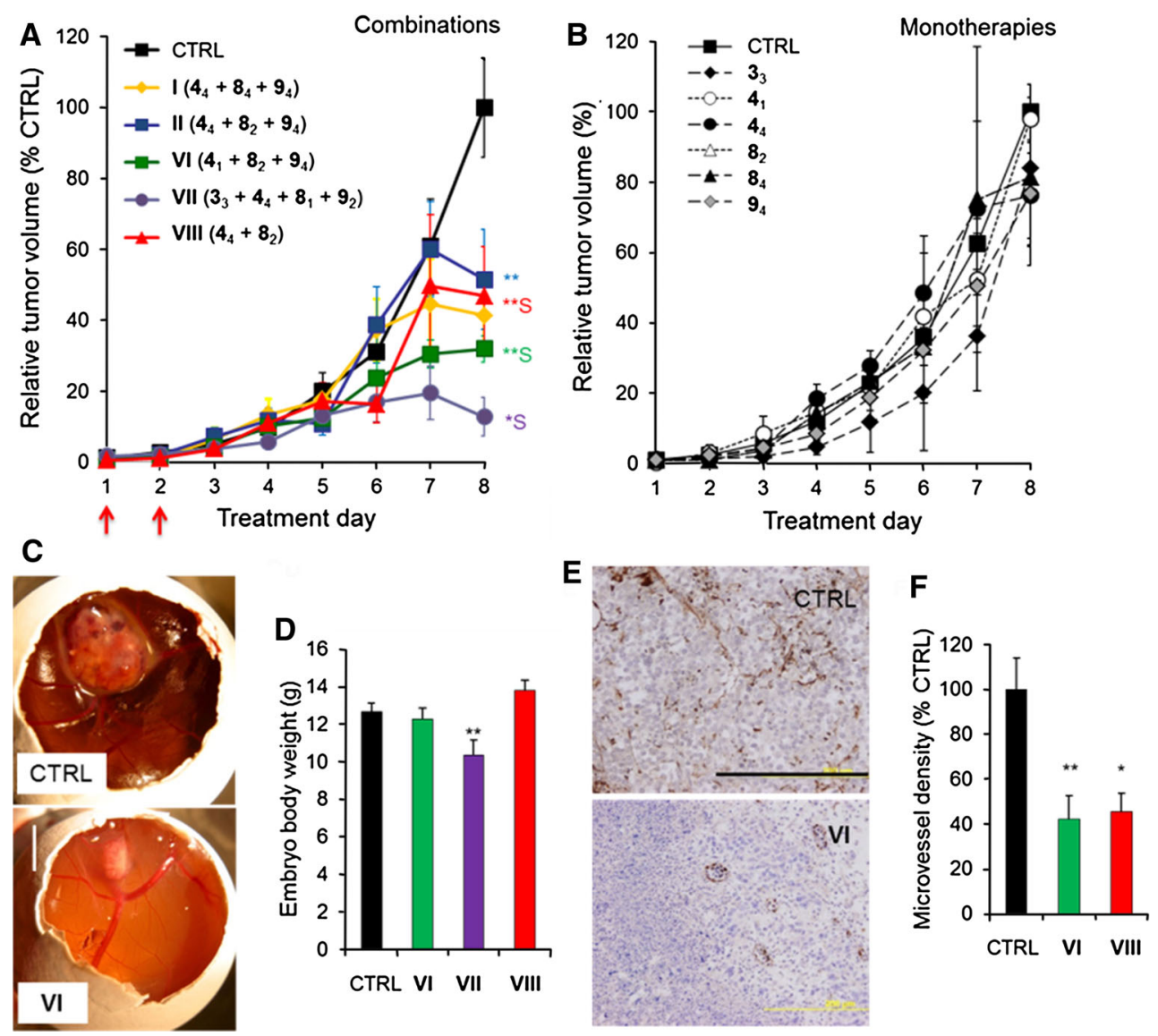

Fig. 4 Inhibition of A2780 tumor growth on the CAM by the optimal drug combinations. Growth curves of A2780 tumors grafted on the CAM $(n=10)$ showing tumor volume as a function of treatment day for various drug combinations (a) and single-drug treatments (b). " $S$ " indicates synergy. Compounds were freshly premixed and administered i.v. on treatment days 1 and 2 (red arrows in a). Data points represent the average tumor volume as a percentage of the final control volume per experiment. c Representative images of vehicle (CTRL) and combination VI treated tumors. d Mean embryo body

promising strategy to improve cancer therapy. The FSC technique was used to navigate through the large parametric space of nine compounds, each considered at four doses, aiming for an optimal angiostatic drug combination. Using a simple in vitro endothelial cell (EC) viability bioassay as the output, an optimal low-dose drug combinations containing axitinib (3), erlotinib (4), RAPTA-C (8) and BEZ-235 (9) was found. The most efficient of these combinations was also effectively inhibiting cancer in two in vivo animal models. We observed that (1) while some drugs showed synergistic interactions, others showed additive or even antagonistic behavior, (2) the observed synergy was drug dose ratio dependent, (3) the combination of angiostatic drugs enhanced endothelial specificity, (4) screening on EC migration did not identify highly efficient drug combinations, and (5) in vitro optimized anti-

weight on the last experiment day for selected treatment groups. e Representative images of CD31-stained tumor sections are shown. The bar in the lower right of the image represents $0.2 \mathrm{~mm}$. The whole image was linearly adjusted for brightness and contrast. f Microvessel density quantification measured as the number of vessels per $\mathrm{mm}^{2}$ and represented as a percentage of the control (CTRL). * indicates $p$ value $<0.05$ and $* *$ indicates $p$ value $<0.01$ student's $t$ test. Error bars represent the SEM. $N=3$ for condition $4_{1} . N=5-11$ for all other groups. (Color figure online)

angiogenic drug combinations translated to anti-angiogenic anticancer effects in vivo.

We previously demonstrated that multi-drug effects can be expressed by a quadratic relationship of the drug-drug interactions [42], which was confirmed in bacterial systems [43]. Here, we have further demonstrated that the response surface for the whole range of drugs and drug doses applied can be expressed as a second-order equation that can be used to formulate optimal drug combinations. The results of this regression modeling (Supplementary Methods) permitted us to eliminate sunitinib (6) a compound which is known to have a similar target profile as axitinib (3) (note that both inhibit signaling of VEGF and PDGF [44]). The exclusion of sunitinib over axitinib appears justifiable, as axitinib is known to be a more selective TKI with stronger affinity for the same targets [44]. Similarly, the exclusion 

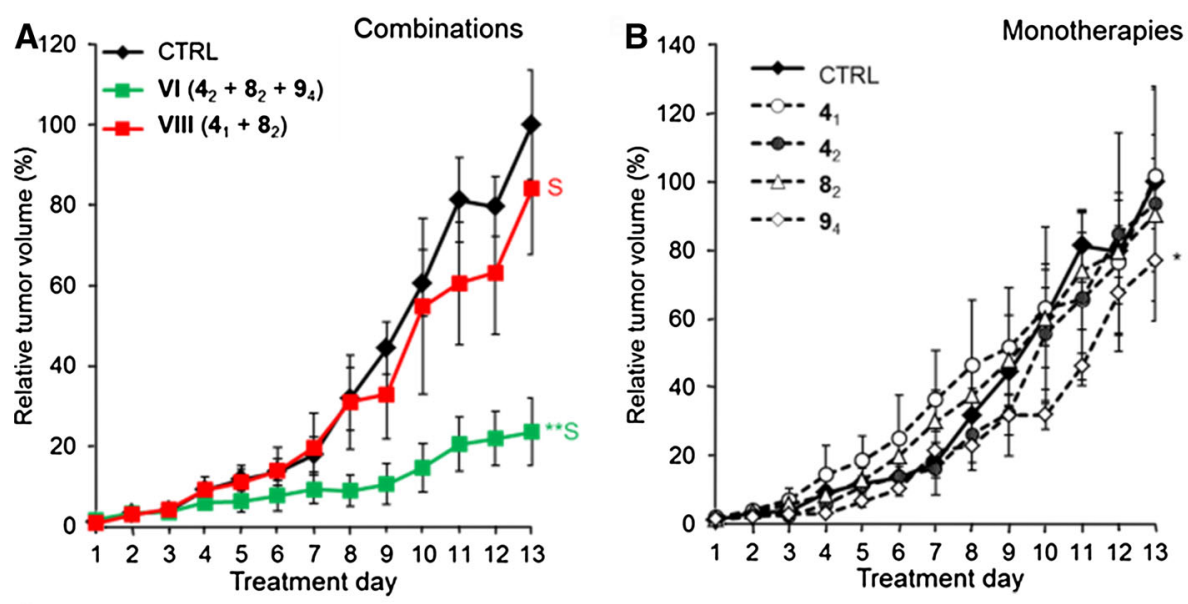

C

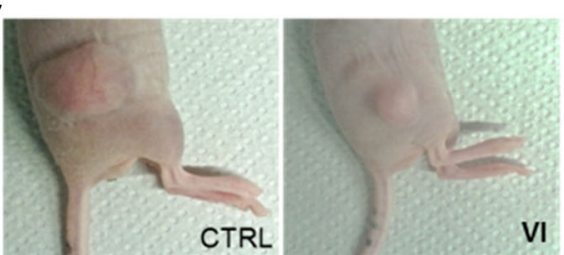

D
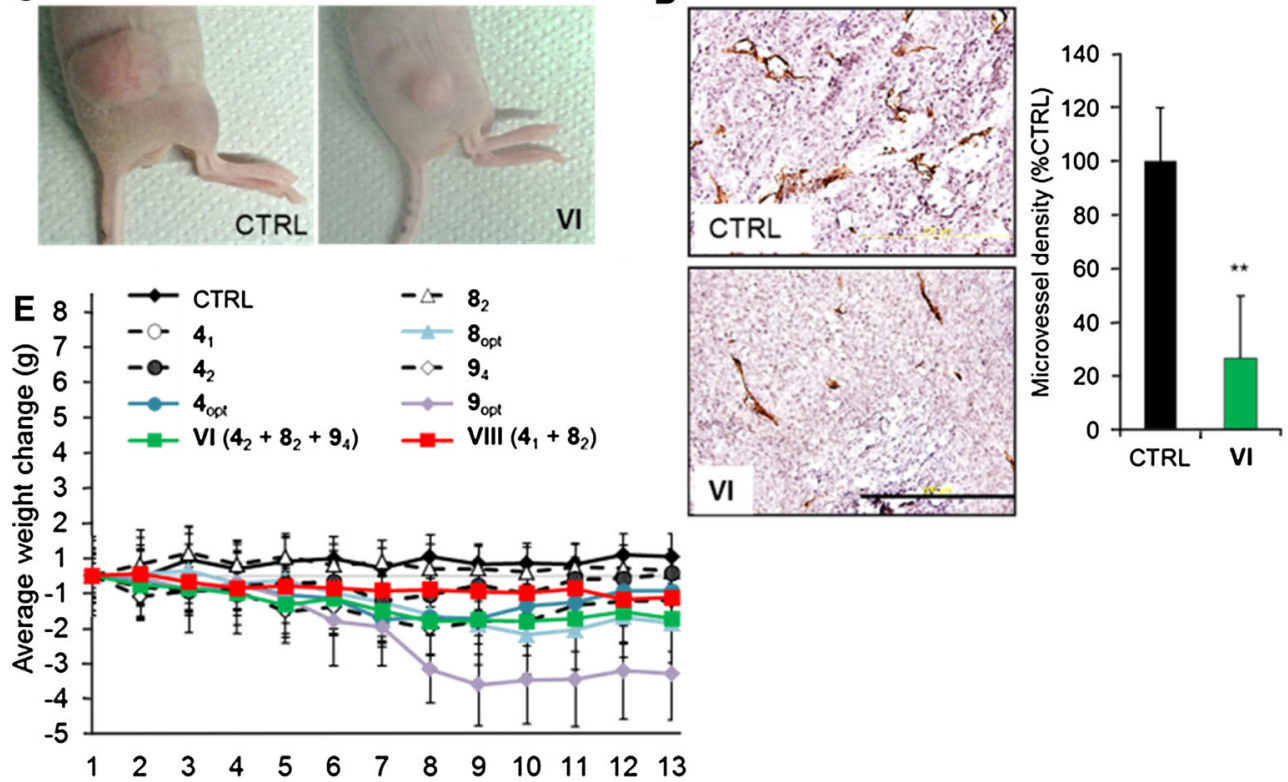

Fig. 5 Inhibition of LS174T tumor growth in athymic mice by the optimal drug combinations. a LS174T tumors grafted subcutaneously in athymic Swiss nu/nu mice and treated daily with the drug combinations as listed in Table 2. b Inhibition of tumor growth by single compounds at indicated doses. Data points represent the average tumor volume as a percentage of the final CTRL volume per experiment, and error bars represent the SEM; $N=3-9$. $* p<0.05$ and $* * p<0.01$ (two-way ANOVA). " $S$ " indicates synergy $(\mathrm{CI}<1)$. c Representative images of vehicle-treated (CTRL) and tumors treated with drug combination VI on the last experiment day.

of bevacizumab (2) was expected, as it is known that EC does not use VEGF as an autocrine growth factor, and tumor angiogenesis is mainly driven by tumor produced VEGF [10].

The four drugs with significant inhibitory single-drug linear contributions to cell viability were compounds axitinib, erlotinib, RAPTA-C and BEZ-235 (Fig. 1d). In terms of intracellular signaling, this combination of drugs appears to make sense in retrospect. EGFR targeting by erlotinib and VEGFR targeting by axitinib result in inhibition of two d Representative images of immunohistochemical staining for the EC marker CD31 and corresponding quantification of microvessel density, measured as the number of vessels per $\mathrm{mm}^{2}$ and presented as a percentage of the CTRL. Results show significantly reduced microvessel density in tumors treated with VI. The bar in the lower panel image represents $0.2 \mathrm{~mm}$ and is valid for both images. The whole images were linearly adjusted for brightness and contrast. e Body weight change during the experiment. $* p<0.05$ and ** $p<0.01$ (student $t$ test). 4 (erlotinib), 8 (RAPTA-C) and 9 (BEZ235). The error bars represent the SEM. (Color figure online)

largely synergistic and widely used cellular signaling pathways, i.e., the PI3K/AKT/mTOR and the ras/raf/MEK/ MAPK signaling pathway, respectively. Since mTORC1 and mTORC2 belong to the PI3K/AKT pathway, one would expect that both signaling pathways are inhibited by EGFR and VEGFR inhibitors. It is also expected that a drug that targets histone proteins, such as RAPTA-C [45], can reinforce the angiostatic effect, as intervention with histone-DNA interactions is known to be angiostatic from the many reports on histone deacetylase inhibitors [46-48]. 
mTOR and EGFR inhibitors have already been identified as a synergistic combination in various cancer cell types $[49,50]$, despite clinically observed toxicity [51, 52].

Based on the analysis of the response surfaces of the second-order linear regression model generated from the four-drug optimization data (Fig. 2c) and embryo weight loss observed in the CAM model (Fig. 4d), axitinib was eliminated from further investigation. Thus, the optimal drug combination containing erlotinib, RAPTA-C and BEZ-235 was identified. It allowed for dose reductions of 5-, 11- and 6-fold, respectively, as compared to the equivalent single-drug dose efficiencies in vitro. Interestingly, enhanced EC specificity was observed for the combinations when compared to the individual compounds. This is another indication that the parallel blocking of multiple angiogenesis pathways can result in synergism for the angiostatic outcome. Simultaneous targeting of different signaling pathways may limit the probability of cells to develop acquired resistance [16].

The migration-based optimization screen failed to reach effective combinations (Supplementary Fig. S3B). This may suggest that proliferation is more dominant in the process of angiogenesis than cell migration, which has also been proposed by others [53]. The same may also be reflected by clinical trials, where proliferation inhibitors (such as sunitinib and BEZ-235) were more successful than migration inhibitors (the $\alpha_{\mathrm{v}} \beta_{3}$ inhibitor cilengitide [54] and the $\alpha_{5} \beta_{1}$ antibody volociximab [55]). Another possible explanation for enhanced success with the proliferation assay over the migration assay may be the selective nature of synergistic drug interactions. As seen by Lehar et al. [15], drug combinations could attain greater selectivity. They suggested that "synergistic combinations tend to be more specific to particular cellular phenotypes than are single drugs." This may explain the preferred success of the screen through selection on basis of proliferation, rather than cell migration.

The optimal drug mixture inhibited tumor growth by approximately $80 \%$, most likely by an inhibitory effect on angiogenesis. Although the detailed mechanism of combination therapy still needs to be understood, the induction of apoptosis as well as the inhibition of tip cells shows part of the effector mechanism. Targeting of tip cells may be another attractive strategy as these cells are indispensable for sprouting angiogenesis. The results provide a promising option for future clinical anti-angiogenic applications.

One might expect that the differences in pharmacokinetics between the components of the drug mixture may interfere with obtaining good results in vivo. Our results imply that (1) the best drug combinations found show smooth response surfaces (Fig. 2c, i.e., moderate changes in drug ratios do not significantly change the output), (2) response surfaces, giving a mathematical description of the magnitude of the interaction for all drug pairs, confirmed in vivo treatment outcome (compare Figs. 2c, 5a), and (3) EC viability observed in vitro seems to be a relatively good parameter for translation to vascular density reduction and tumor growth inhibition in vivo.

The current study shows that FSC applied in vitro can be used for the fast and reliable identification of potent, lowdose angiostatic drug combinations in vivo. It is likely that combining the optimal anti-angiogenic compounds with other treatment strategies may lead to even better cancer treatment outcomes. The capacity to normalize the tumor vasculature provides angiostatic strategies with outstanding combination therapy features $[56,57]$. The impact of the method also lies in the fact that it can be applied in a variety of situations, e.g., for finding drug mixtures directly targeting tumor cells. This strategy would then also offer the opportunity for a personalized approach, by performing a drug screen on freshly isolated tumor cells from a patient biopsy. Such strategy would also depend on faster screening methods. We are currently working on improvement of the FSC method in order to make the selection procedure faster. In conclusion, designing effective, synergistic and specific multi-component drug combinations may become a key approach in developing new therapies for cancer and other diseases.

Acknowledgments We would like to thank Prof. Dean Ho (UCLA) and Prof. Michel Aguet (EPFL) for critically reviewing the manuscript. The project is partially supported by Swiss Federal Institute of Technology (EPFL to PNS), Center for Translational Molecular Medicine (CTMM to AWG), The Netherlands, European Union (PIEF-GA-2013-626797 to PNS), Union for International Cancer Control (ICRET-13-080 to PNS), and the National Natural Science Foundation of China (81301293 to XD).

Conflict of interest The authors declare that they have no competing interests.

Open Access This article is distributed under the terms of the Creative Commons Attribution License which permits any use, distribution, and reproduction in any medium, provided the original author(s) and the source are credited.

\section{References}

1. Folkman J (1971) Tumor angiogenesis: therapeutic implications. N Engl J Med 285(21):1182-1186

2. Carmeliet P, Jain RK (2000) Angiogenesis in cancer and other diseases. Nature 407(6801):249-257

3. Gonzalez-Angulo AM, Hortobagyi GN, Ellis LM (2011) Targeted therapies: peaking beneath the surface of recent bevacizumab trials. Nat Rev Clin Oncol 8(6):319-320

4. Gerlinger M, Rowan AJ, Horswell S, Larkin J, Endesfelder D, Gronroos E, Martinez P, Matthews N, Stewart A, Tarpey P, Varela I, Phillimore B, Begum S, McDonald NQ, Butler A, Jones D, Raine K, Latimer C, Santos CR, Nohadani M, Eklund AC, 
Spencer-Dene B, Clark G, Pickering L, Stamp G, Gore M, Szallasi Z, Downward J, Futreal PA, Swanton C (2012) Intratumor heterogeneity and branched evolution revealed by multiregion sequencing. N Engl J Med 366(10):883-892

5. Hoey T (2010) Drug resistance, epigenetics, and tumor cell heterogeneity. Sci Transl Med 2(28):28ps19

6. Hayman SR, Leung N, Grande JP, Garovic VD (2012) VEGF inhibition, hypertension, and renal toxicity. Curr Oncol Rep 14 (4):285-294

7. Ebos JM, Lee CR, Cruz-Munoz W, Bjarnason GA, Christensen JG, Kerbel RS (2009) Accelerated metastasis after short-term treatment with a potent inhibitor of tumor angiogenesis. Cancer Cell 15(3):232-239

8. Loges S, Schmidt T, Carmeliet P (2010) Mechanisms of resistance to anti-angiogenic therapy and development of thirdgeneration anti-angiogenic drug candidates. Genes Cancer 1 (1): $12-25$

9. van Beijnum JR, Nowak-Sliwinska P, Huijbers EJ, Thijssen VL, Griffioen AW (2015) The great escape; the hallmarks of resistance to anti-angiogenic therapy. Pharmacol Rev 67:1-21

10. Griffioen AW, Molema G (2000) Angiogenesis: potentials for pharmacologic intervention in the treatment of cancer, cardiovascular diseases, and chronic inflammation. Pharmacol Rev 52 (2):237-268

11. Lignet F, Calvez V, Grenier E, Ribba B (2013) A structural model of the VEGF signalling pathway: emergence of robustness and redundancy properties. Math Biosci Eng 10(1):167-184

12. Griffioen AW, Weiss A, Berndsen RH, Abdul UK, Te Winkel MT, Nowak-Sliwinska P (2014) The emerging quest for the optimal angiostatic combination therapy. Biochem Soc Trans 42 (6): 1608-1615

13. Misale S, Arena S, Lamba S, Siravegna G, Lallo A, Hobor S, Russo M, Buscarino M, Lazzari L, Sartore-Bianchi A, Bencardino K, Amatu A, Lauricella C, Valtorta E, Siena S, Di Nicolantonio F, Bardelli A (2014) Blockade of EGFR and MEK intercepts heterogeneous mechanisms of acquired resistance to anti-EGFR therapies in colorectal cancer. Sci Transl Med 6 (224):224ra226

14. Sen B, Peng S, Tang X, Erickson HS, Galindo H, Mazumdar T, Stewart DJ, Wistuba I, Johnson FM (2012) Kinase-impaired BRAF mutations in lung cancer confer sensitivity to dasatinib. Sci Transl Med 4(136):136ra170

15. Lehar J, Krueger AS, Avery W, Heilbut AM, Johansen LM, Price ER, Rickles RJ, Short GF 3rd, Staunton JE, Jin X, Lee MS, Zimmermann GR, Borisy AA (2009) Synergistic drug combinations tend to improve therapeutically relevant selectivity. Nat Biotechnol 27(7):659-666

16. Bozic I, Reiter JG, Allen B, Antal T, Chatterjee K, Shah P, Moon YS, Yaqubie A, Kelly N, Le DT, Lipson EJ, Chapman PB, Diaz LA Jr, Vogelstein B, Nowak MA (2013) Evolutionary dynamics of cancer in response to targeted combination therapy. Elife 2: e00747

17. Sequist LV, Waltman BA, Dias-Santagata D, Digumarthy S, Turke AB, Fidias P, Bergethon K, Shaw AT, Gettinger S, Cosper AK, Akhavanfard S, Heist RS, Temel J, Christensen JG, Wain JC, Lynch TJ, Vernovsky K, Mark EJ, Lanuti M, Iafrate AJ, Mino-Kenudson M, Engelman JA (2011) Genotypic and histological evolution of lung cancers acquiring resistance to EGFR inhibitors. Sci Trans Med 3(75):75ra26

18. Feldman DR, Baum MS, Ginsberg MS, Hassoun H, Flombaum CD, Velasco S, Fischer P, Ronnen E, Ishill N, Patil S, Motzer RJ (2009) Phase I trial of bevacizumab plus escalated doses of sunitinib in patients with metastatic renal cell carcinoma. J Clin Oncol 27(9):1432-1439

19. Spreafico A, Chi KN, Sridhar SS, Smith DC, Carducci MA, Kavsak P, Wong TS, Wang L, Ivy SP, Mukherjee SD,
Kollmannsberger CK, Sukhai MA, Takebe N, Kamel-Reid S, Siu LL, Hotte SJ (2014) A randomized phase II study of cediranib alone versus cediranib in combination with dasatinib in docetaxel resistant, castration resistant prostate cancer patients. Invest New Drugs 32(5):1005-1016

20. Mayer LD, Janoff AS (2007) Optimizing combination chemotherapy by controlling drug ratios. Mol Interv 7(4):216-223

21. Pritchard JR, Lauffenburger DA, Hemann MT (2012) Understanding resistance to combination chemotherapy. Drug Resist Update 15(5-6):249-257

22. Wong PK, Yu F, Shahangian A, Cheng G, Sun R, Ho CM (2008) Closed-loop control of cellular functions using combinatory drugs guided by a stochastic search algorithm. Proc Natl Acad Sci 105(13):5105-5110

23. Price KV, Storn RM, Lampinen JA (2005) Differential evolution: a practical approach to global optimization. Natural computing series. Springer, Berlin

24. Calzolari D, Bruschi S, Coquin L, Schofield J, Feala JD, Reed JC, McCulloch AD, Paternostro G (2008) Search algorithms as a framework for the optimization of drug combinations. PLoS Comput Biol 4(12):e1000249

25. Dancey JE, Chen HX (2006) Strategies for optimizing combinations of molecularly targeted anticancer agents. Nat Rev Drug Discov 5(8):649-659

26. Feala JD, Cortes J, Duxbury PM, Piermarocchi C, McCulloch AD, Paternostro G (2010) Systems approaches and algorithms for discovery of combinatorial therapies. Wiley Interdiscip Rev Syst Biol Med 2(2):181-193

27. Park M, Nassar M, Vikalo H (2013) Bayesian active learning for drug combinations. IEEE Trans Biomed Eng 60(11):3248-3255

28. Marshall J (2006) Clinical implications of the mechanism of epidermal growth factor receptor inhibitors. Cancer 107(6):12071218

29. Adhireksan Z, Davey GE, Campomanes P, Groessl M, Clavel CM, Yu H, Nazarov AA, Yeo HF, Ang WH, Droge P, Rothlisberger U, Dyson PJ, Davey CA (2014) A ligand distinction between two ruthenium-cymen anticancer agents determines protein versus DNA targeting. Nat Commun 5:3462

30. Garber K (2009) Targeting mTOR: something old, something new. J Natl Cancer Inst 101(5):288-290

31. van Beijnum JR, Nowak-Sliwinska $P$, van den Boezem E, Hautvast P, Buurman WA, Griffioen AW (2013) Tumor angiogenesis is enforced by autocrine regulation of high-mobility group box 1. Oncogene 17(32):363-374

32. Siemerink MJ, Klaassen I, Vogels IM, Griffioen AW, Van Noorden CJ, Schlingemann RO (2012) CD34 marks angiogenic tip cells in human vascular endothelial cell cultures. Angiogenesis 15 (1):151-163

33. Nowak-Sliwinska $P$, van Beijnum JR, van Berkel M, van den Bergh H, Griffioen AW (2010) Vascular regrowth following photodynamic therapy in the chicken embryo chorioallantoic membrane. Angiogenesis 13(4):281-292

34. Tsutsui H, Valamehr B, Hindoyan A, Qiao R, Ding X, Guo S, Witte ON, Liu X, Ho CM, Wu H (2011) An optimized small molecule inhibitor cocktail supports long-term maintenance of human embryonic stem cells. Nat Commun 2:167

35. Yu F, Al-Shyoukh I, Feng J, Li X, Liao CW, Ho CM, Shamma JS, Sun R (2011) Control of Kaposi's sarcoma-associated herpesvirus reactivation induced by multiple signals. PLoS One 6(6): e20998

36. Weiss A, van Beijnum JR, Bonvin D, Jichlinski P, Dyson PJ, Griffioen AW, Nowak-Sliwinska P (2014) Low-dose angiostatic tyrosine kinase inhibitors improve photodynamic therapy for cancer: lack of vascular normalization. J Cell Mol Med 18:480-491

37. Ding X, Sanchez DJ, Shahangian A, Al-Shyoukh I, Cheng G, Ho CM (2012) Cascade search for HSV-1 combinatorial drugs with 
high antiviral efficacy and low toxicity. Int J Nanomed 7:22812292

38. Stanescu R, Didilescu AC, Jianu AM, Rusu MC (2012) Angiogenesis in the reparatory mucosa of the mandibular edentulous ridge is driven by endothelial tip cells. Rom J Morphol Embryol 53(2):375-378

39. Vrapciu AD, Rusu MC, Voinea LM (2014) Immunohistochemistry of a choroidal melanoma: nestin, CD34 and CD117/c-kit labeling. Rom J Morphol Embryol 55(2):437-442

40. Reuwer AQ, Nowak-Sliwinska P, Mans LA, van der Loos CM, von der Thüsen JH, Twickler MT, Spek CA, Goffin V, Griffioen AW, Borensztajn KS (2012) Functional consequences of prolactin signaling in endothelial cells: A potential link with angiogenesis in pathophysiology? J Cell Mol Med 16(9):20352048

41. Weiss A, Berndsen BH, Dubois M, Müller M, Schibli R, Griffioen AW, Dyson PJ, Nowak-Sliwinska P (2014) In vivo antitumor activity of the organometallic ruthenium(II)-arene complex [Ru(n6-p-cymene)Cl2(pta)] (RAPTA-C) in human ovarian and colorectal carcinomas. Chem Sci 5:4742-4748

42. Al-Shyoukh I, Yu F, Feng J, Yan K, Dubinett S, Ho CM, Shamma JS, Sun R (2011) Systematic quantitative characterization of cellular responses induced by multiple signals. BMC Syst Biol 5:88

43. Wood K, Nishida S, Sontag ED, Cluzel P (2012) Mechanismindependent method for predicting response to multidrug combinations in bacteria. Proc Natl Acad Sci 109(30):12254-12259

44. Bhargava P, Robinson MO (2011) Development of second-generation VEGFR tyrosine kinase inhibitors: current status. Curr Oncol Rep 13(2):103-111

45. Adhireksan Z, Davey GE, Campomanes P, Groessl M, Clavel $\mathrm{CM}$, Yu H, Nazarov AA, Yeo CH, Ang WH, Droge P, Rothlisberger U, Dyson PJ, Davey CA (2014) Ligand substitutions between ruthenium-cymene compounds can control protein versus DNA targeting and anticancer activity. Nat Commun 5:3462

46. Martin M, Potente M, Janssens V, Vertommen D, Twizere JC, Rider MH, Goris J, Dimmeler S, Kettmann R, Dequiedt F (2008) Protein phosphatase 2A controls the activity of histone deacetylase 7 during $\mathrm{T}$ cell apoptosis and angiogenesis. Proc Natl Acad Sci 105(12):4727-4732

47. Hellebrekers DM, Castermans K, Vire E, Dings RP, Hoebers NT, Mayo KH, Oude Egbrink MG, Molema G, Fuks F, van Engeland M, Griffioen AW (2006) Epigenetic regulation of tumor endothelial cell anergy: silencing of intercellular adhesion molecule- 1 by histone modifications. Cancer Res 66(22):10770-10777
48. Rossig L, Li H, Fisslthaler B, Urbich C, Fleming I, Forstermann U, Zeiher AM, Dimmeler S (2002) Inhibitors of histone deacetylation downregulate the expression of endothelial nitric oxide synthase and compromise endothelial cell function in vasorelaxation and angiogenesis. Circ Res 91(9):837-844

49. Bianco R, Garofalo S, Rosa R, Damiano V, Gelardi T, Daniele G, Marciano R, Ciardiello F, Tortora G (2008) Inhibition of mTOR pathway by everolimus cooperates with EGFR inhibitors in human tumours sensitive and resistant to anti-EGFR drugs. Br J Cancer 98(5):923-930

50. Rao RD, Mladek AC, Lamont JD, Goble JM, Erlichman C, James CD, Sarkaria JN (2005) Disruption of parallel and converging signaling pathways contributes to the synergistic antitumor effects of simultaneous mTOR and EGFR inhibition in GBM cells. Neoplasia 7(10):921-929

51. Bauman JE, Arias-Pulido H, Lee SJ, Fekrazad MH, Ozawa H, Fertig E, Howard J, Bishop J, Wang H, Olson GT, Spafford MJ, Jones DV, Chung CH (2013) A phase II study of temsirolimus and erlotinib in patients with recurrent and/or metastatic, platinum-refractory head and neck squamous cell carcinoma. Oral Oncol 49(5):461-467

52. Wen PY, Chang SM, Lamborn KR, Kuhn JG, Norden AD, Cloughesy TF, Robins HI, Lieberman FS, Gilbert MR, Mehta MP, Drappatz J, Groves MD, Santagata S, Ligon AH, Yung WK, Wright JJ, Dancey J, Aldape KD, Prados MD, Ligon KL (2014) Phase I/II study of erlotinib and temsirolimus for patients with recurrent malignant gliomas: North American Brain Tumor Consortium trial 04-02. Neuro-oncol 16(4):567-578

53. Hinow P, Gerlee P, McCawley LJ, Quaranta V, Ciobanu M, Wang S, Graham JM, Ayati BP, Claridge J, Swanson KR, Loveless M, Anderson AR (2009) A spatial model of tumor-host interaction: application of chemotherapy. Math Biosci Eng 6 (3):521-546

54. Reardon DA, Cheresh D (2011) Cilengitide: a prototypic integrin inhibitor for the treatment of glioblastoma and other malignancies. Genes Cancer 2(12):1159-1165

55. Almokadem S, Belani CP (2012) Volociximab in cancer. Expert Opin Biol Ther 12(2):251-257

56. Jain RK (2005) Normalization of tumor vasculature: an emerging concept in antiangiogenic therapy. Science 307(5706):58-62

57. Weiss A, Bonvin D, Berndsen B, Sherrer E, Dyson PJ, Griffioen AW, Nowak-Sliwinska P (2015) Angiostatic treatment prior to chemo- or photodynamic therapy improves anti-tumor efficacy. Sci Rep 5(8990):1-8 